EPJ Web of Conferences 73, 02011 (2014)

DOI: $10.1051 /$ epjconf/20147302011

(C) Owned by the authors, published by EDP Sciences, 2014

\title{
Generalized loop space and TMDs
}

\author{
Tom Mertens ${ }^{\mathrm{a}}$ \\ Universiteit Antwerpen, Groenenborgerlaan 171, 2020 Antwerpen, Belgium
}

\begin{abstract}
The Standard Model describes the three (of four) basic interactions known in Nature in terms of the quantum fields which are constituted by representations of special unitary gauge groups of symmetry. However, the physical observables do not always coincide with the fundamental degrees of freedom of the Standard Model. Therefore it can be useful to switch to the loop space representation of the gauge theory, where the variables are inherently gauge invariant but the degrees of freedom are absorbed in the path/loop dependence. Over-completeness of this space requires the introduction of an equivalence relation which is provided by Wilson loop functionals operating on piecewise regular paths. It is well known that certain Wilson loops show the same singularity structure as some Transverse Momentum Dependent PDFs (TMDs), which are not renormalizable by the common methods due to exactly this singularity structure. By introducing geometrical operators, like the area-derivative, we were able to derive an evolution equation for these Wilson loops and we hope to apply this method in the future to find some renormalization schemes for TMDs.
\end{abstract}

\section{Introduction}

In current nuclear research a lot of effort is directed at understanding and determining the threedimensional structure of nucleons. To investigate this complicated internal structure several new functions have been put forward in theory, which all try to describe some aspects of this structure. Among these functions there are the Transverse Momentum Dependent Parton Distribution Functions (TMDs), used to describe the transverse motion of the partons in nucleons. Although there is currently no global consensus on the exact correct definition, we will here restrict ourselves to the operator definition [1-4]. This definition depends on the correlator:

$$
\Phi_{s s^{\prime}}^{\Gamma}\left(x, \vec{k}_{\perp}, \mu\right)=\frac{1}{2} \int \frac{d z^{-} d^{2} z_{\perp}}{(2 \pi)\left(2 \pi^{2}\right)} e^{i k \cdot z}\left\langle P, s\left|\overline{\psi_{i}}(0) \Gamma_{i j} \mathcal{W}_{\mathrm{TMD}} \psi_{j}(z)\right| P, s^{\prime}\right\rangle_{z^{+}=0},
$$

where for twist-2 operators $\Gamma=\gamma^{+}, \gamma^{+} \gamma^{5}, i \sigma^{i+} \gamma^{5}$ and for twist-3 $\Gamma=\gamma^{i}$. $\mathcal{W}_{\text {TMD }}$ represents the Wilson line structure of the TMD (for details see for instance [3]). Unfortunately quantum corrections in

\footnotetext{
ae-mail: tom.mertens@uantwerpen.be
}

This is an Open Access article distributed under the terms of the Creative Commons Attribution License 4.0, which permits unrestricted use, distribution, and reproduction in any medium, provided the original work is properly cited. 
this correlator give rise to overlapping light-cone and rapidity divergencies, resulting in problems for renormalizability and evolution of TMDs.

On the other hand in [5-10] it was demonstrated that there exists a duality between light-like npolygonal Wilson loops and n-gluon scattering amplitudes in $\mathcal{N}=4$ Super-Yang-Mills (SYM) theory. These polygonal loops have a specific parametrization in dual coordinates $x_{i}$ related to the momenta of the gluons in the following way: $p_{i}=x_{i}-x_{i+1}$, the $p_{i}$ representing the gluon momenta. A very interesting feature of this duality is that the infrared (IR) divergencies of the gluon scattering amplitudes, for which we know their cut-off is related to the cusp anomalous dimension [11-13], are mapped to the ultraviolet (UV) divergences of the corresponding Wilson loop.

The connection with TMDs is made by the observation that the singularity structure of TMDs is captured by its Wilson line structure such that it is not a big surprise that TMDs show similar singularity behavior to certain classes of Wilson loops. The above observations motivated us to study a loop space constructed of Wilson loops and allowed us to conjecture an evolution equation for TMDs defined by making use of the correlator (1), where the area variation of the Wilson loops is mapped to the rapidity evolution of the corresponding TMDs [14].

\section{Generalized loop space}

In the Ambrose-Singer theorem [15] it is demonstrated that the information captured by a gauge theory can also be described in a gauge invariant way by considering the traces of holonomies of that theory, naturally introducing a loop space representation of a gauge theory. A problem with this approach is that when one considers a naive loop space representation this leads to some problems [16]:

(i) Reparametrization group: are loops with different parametrization considered equal or not?

(ii) Over-completeness: the information is covered more than once by the holonomy data.

(iii) Trace constraints: they assure that some value can be written as the trace of a holonomy.

(iv) Mandelstam constraints (infinitely many): combinations of holonomies can be written as a holonomy over some loop.

The solution we used to assess these problems is to use the generalized loop space introduced in [17], which makes use of Chen's algebraic paths [18]. Here the usual loop space is endowed with an extra equivalence relation realized by the Wilson loop functionals:

$$
\mathcal{W}[C]=\frac{1}{N_{c}} \operatorname{Tr}\left\langle 0\left|\mathcal{P} e^{i g \oint d z^{\mu} A_{\mu}^{a}(z) t_{a}}\right| 0\right\rangle, \quad \mathcal{C}: \quad z^{\mu}(s) \quad s=0 \ldots 1 \quad \text { where } \quad z^{\mu}(0) \equiv z^{\mu}(1),
$$

but where loops are now generalized using Chen's iterated integrals [18, 19]:

$$
X^{\omega_{1} \cdots \omega_{r}}(\mathcal{C})=\int_{\mathcal{C}} \omega_{1} \cdots \omega_{r}=\int_{0}^{1}\left(\int_{\mathcal{C}^{t}} \omega_{1} \cdots \omega_{r-1}\right) \omega_{r}(t) d t .
$$

Since line integrals are reparametrization invariant the reparametrization problem is solved. Moreover the Wilson loop functionals can now be expressed using Chen's integrals:

$$
\operatorname{Tr}\left[U_{\Gamma}\right]=\operatorname{Tr}[1]+\operatorname{Tr}\left[\int_{\Gamma} \omega\right]+\operatorname{Tr}\left[\int_{\Gamma} \omega_{1} \omega_{2}\right]+\cdots .
$$

It can be shown that this equivalence relation also takes care of the trace problem, the over-completeness and the Mandelstam constraints due to the elegant mathematical structure of the space of generalized loops. The loop space can be turned, using the Gel'fand spectrum, into a topological group which is Banach, Hopf, Hausdorff, Tychonov, commutative, nuclear and multiplicative convex. In [17] it is shown 
that its tangent space can be identified with an (infinite dimensional) Lie Algebra structure allowing for different differential operators.

\section{Differential operators}

Among the differential operators that are allowed on generalized loop space we have the path- and areaderivatives [17, 20, 21]. Both of them were used by Makeenko and Migdal in combination with the Dyson-Schwinger approach to derive their Makeenko-Migdal loop equations:

$$
\left\langle\frac{1}{N_{c}} \operatorname{Tr} \mathcal{P} e^{\oint_{\Gamma} d z^{\mu} \mathcal{A}_{\mu}}\right\rangle=\frac{g^{2} N_{c}}{2} \oint_{\Gamma} d y_{\nu} \delta^{n}(x-y)\left(\left\langle\phi\left(\Gamma_{y x}\right) \phi\left(\Gamma_{x y}\right)\right\rangle-\frac{1}{N_{c}^{2}}\langle\phi(\Gamma)\rangle .\right)
$$

Unfortunately the area-derivative they used is in a sense unaware of the cusps along the path. Not surprisingly when one applies this area-derivative to a cusped contour like the light-like polygons this leads to problems. More specifically, when considering a planar rectangle on the light-cone, this leads to the emergence of extra divergences which cannot be handled by the regular regularization schemes. This lead us to introduce a new differential operator, which for the specific Wilson loop rectangle on the light-cone is explicitly given by [22-26]:

$$
\frac{d}{d \ln \sigma}=s \frac{\delta}{\delta s}+t \frac{\delta}{\delta t}, \quad s=\left(l_{1}+l_{2}\right)^{2}, t=\left(l_{1}+l_{3}\right)^{2}
$$

and where $l_{i}^{2}=0$ since they are considered to lie on the light-cone.

\section{Conjectured evolution equation}

When we apply the above defined differential operator in combination with the usual renormalization mass scale differential operator $\frac{d}{d \ln \mu}$ to the leading order result of the rectangular Wilson loop on the light-cone $[27,28]$ :

$$
W_{\text {L.O. }}\left(\Gamma_{\square}\right)=1-\frac{\alpha_{s} C_{F}}{\pi}\left(2 \pi \mu^{2}\right)^{\epsilon} \Gamma(1-\epsilon)\left[\frac{1}{\epsilon^{2}}\left(-\frac{s}{2}\right)^{\epsilon}+\frac{1}{\epsilon^{2}}\left(-\frac{t}{2}\right)^{\epsilon}-\frac{1}{2}\left(\ln ^{2} \frac{s}{-t}+\pi^{2}\right)\right]+\mathcal{O}\left(\alpha_{s}^{2}\right),
$$

we arrive at the following equation (in the large $N_{c}$ limit) [22-25]:

$$
\mu \frac{d}{d \mu} \frac{\delta \ln W\left(\Gamma_{\square}\right)}{\delta \ln \sigma}=-4 \Gamma_{\text {cusp }}, \Gamma_{\text {cusp }}=\frac{\alpha_{s} N_{c}}{2 \pi}+O\left(\alpha_{s}^{2}\right) .
$$

Here $\Gamma_{\text {cusp }}$ represents the cusp anomalous dimension. This lead us to conjecture an evolution equation for Wilson loop contours with cusps by generalizing (8) for contours with any number of cusps:

$$
\mu \frac{d}{d \mu} \frac{\delta \ln W\left(\Gamma_{\square}\right)}{\delta \ln \sigma}=-\sum_{\text {cusps }} \Gamma_{\text {cusp }}
$$

In [22] we also demonstrated that our conjecture is valid for a Pi-shaped Wilson loop (which closes at infinity) formed by two off-light-cone semi-infinites and a finite segment on the light-cone. Furthermore in [29] we demonstrated the validity, at leading order, of our conjecture for two simple extensions of our originally considered quadrilateral Wilson loops, one with overlapping segments and one with a self-intersection. Recently [30] we also demonstrated that the new derivative we introduced in [22] is a special case of the Fréchet derivative, itself having a perturbative expansion when applied to the lightlike quadrilateral supports our belief that (9) is valid for higher-orders. In [31] we will show explicitly that it is valid for the light-like quadrilateral at next-to-leading order. 


\section{Summary and outlook}

Using a newly introduced differential operator in generalized loop space, a special case of the Fréchet derivative, we were able to conjecture an evolution equation for certain classes of Wilson loops. Some of these loops have a similar singularity structure to TMDs, so we have the hope that this will lead us to an evolution equation for TMDs in the near future. In [31] we will show that our conjecture is also valid at next-to-leading order, moreover we recently discovered that it might even be possible to demonstrate that it its valid to all orders, but this is still under investigation.

\section{References}

[1] A.V. Belitsky, X. Ji, F. Yuan, Nucl. Phys. B 656, 165 (2003), hep-ph/0208038

[2] D. Boer, P. Mulders, F. Pijlman, Nucl. Phys. B 667, 201 (2003), hep-ph/0303034

[3] I. Cherednikov, N. Stefanis, Phys. Rev. D 77, 094001 (2008), 0710.1955

[4] I. Cherednikov, N. Stefanis, Nucl. Phys. B 802, 146 (2008), 0802. 2821

[5] J. Drummond, J. Henn, G. Korchemsky, E. Sokatchev, Nucl. Phys. B 828, 317 (2010), 0807.1095

[6] J. Drummond, J. Henn, V. Smirnov, E. Sokatchev, JHEP 0701, 064 (2007), hep-th/0607160

[7] L.F. Alday, J.M. Maldacena, JHEP 0706, 064 (2007), 0705.0303

[8] Y. Makeenko, JHEP 0301, 007 (2003), hep-th/0210256

[9] G.P. Korchemsky, J.M. Drummond, E. Sokatchev, Nucl. Phys. B 795, 385 (2008), 0707.0243

[10] L.F. Alday, B. Eden, G.P. Korchemsky, J. Maldacena, E. Sokatchev, JHEP 1109, 123 (2011), 1007.3243

[11] G. Korchemsky, A. Radyushkin, Sov. J. Nucl. Phys. 45, 127 (1987)

[12] G. Korchemsky, A. Radyushkin, Sov. J. Nucl. Phys. 44, 877 (1986)

[13] A.M. Polyakov, Nucl. Phys. B 164, 171 (1980)

[14] I.O. Cherednikov, T. Mertens, P. Taels, F.F. Van der Veken, Int. J. Mod. Phys. Conf. Ser. 25, 1460006 (2014), 1308.3116

[15] W. Ambrose, I. Singer, Transactions of the American Mathematical Society 75, 428 (1953)

[16] R. Giles, Phys. Rev. D 24, 2160 (1981)

[17] J.N. Tavares, Int. J. Mod. Phys. A 9, 4511 (1994), 9305173

[18] K.T. Chen, J. Alg. 9, 8 (1968)

[19] K.T. Chen, Tr. Am. Math. Soc. 156, 359 (1971)

[20] Y. Makeenko, A.A. Migdal, Phys. Lett. B 88, 135 (1979)

[21] Y. Makeenko, Non-Perturbative Methods in Gauge Theory, lecture notes (1995)

[22] I. Cherednikov, T. Mertens, F.F. Van der Veken, Phys. Rev. D 86, 085035 (2012), 1208. 1631

[23] I.O. Cherednikov, T. Mertens, F.F. Van der Veken, Int. J. Mod. Phys. Conf. Ser. 20, 109 (2012), 1208.5410

[24] F.F. Van der Veken, I.O. Cherednikov, T. Mertens, AIP Conf. Proc. 1523, 272 (2013), 1212.4345

[25] I.O. Cherednikov, T. Mertens, F. Van der Veken, Phys. Part. Nucl. 44, 250 (2013), 1210. 1767

[26] F.F. Van der Veken (2013), 1302.6765

[27] I.A. Korchemskaya, G.P. Korchemsky, Phys. Lett. B 287, 169 (1992)

[28] J.M. Drummond, J. Henn, G.P. Korchemsky, E. Sokatchev, Nucl. Phys. B 795, 52 (2008)

[29] T. Mertens, P. Taels, Physics Letters B 727, 563 (2013)

[30] I. Cherednikov, T. Mertens (2014), 1401.2721

[31] I. Cherednikov, T. Mertens, In preparation (2014) 